\title{
Veneers in esthetic zone - A case series
}

\author{
Resham Narula ${ }^{1, *}$, Saru Dhir ${ }^{2}$, Saurabh Gupta ${ }^{3}$, Bharat Gupta ${ }^{4}$, Neha Patil ${ }^{5}$, Manjushree Juneja ${ }^{6}$, \\ Saurabh Juneja ${ }^{7}$

\begin{abstract}
${ }^{1}$ PG Student, ${ }^{2,5}$ Senior Lecturer, ${ }^{3}$ Professor, ${ }^{4,6,7}$ Reader, ${ }^{1,3}$ Dept. of Conservative Dentistry and Endodontics, ${ }^{2}$ Dept. of Pedodontics and Preventive Dentistry, ${ }^{4}$ Dept. of Periodontology, ${ }^{5,6}$ Dept. of Oral Medicine and Radiology, ${ }^{7}$ Dept. of Oral Pathology, ${ }^{1,3}$ D.A.V (C) Dental College and Hospital, Yamunanagar, Haryana, ${ }^{2}$ MM College of Dental Sciences, Mullana, Haryana, ${ }^{4,5}$ MGM Dental College, Navi Mumbai, Maharashtra, ${ }^{6,7}$ ITS Dental College, Murad Nagar, Ghaziabad, Uttar Pradesh, India
\end{abstract}

*Corresponding Author:

Email: resham.narula@gmail.com

\begin{abstract}
With the growth of cosmetic dental procedures, the practise of dentistry has become increasingly centred on fulfilling patient desires. Porcelain veneers are increasing in popularity among today's patients and dental practitioners for conservative restoration of unaesthetic anterior teeth. The ability to place restorations without significant removal of structure is becoming more critical than ever. Besides being painless and conservative, it is a durable material and doesn't stain like composite making it attractive for a much longer period of time thus making contemporary dentistry, increasingly oriented toward elective and cosmetic procedures.
\end{abstract}

Keywords: Veneers, Esthetics, Conservative preparation, Porcelain.

\section{Introduction}

One of the fascinating findings in psychological research in the past one decade has been the existence of physical attraction stereotype. With media sovereigning the world, the striking theme of "beautiful is always better" has taken a front seat. This is especially characteristic among the younger generation which besides being highly competitive weighs an attractive personality in the same balancing scale because of which the latest technologies are based on the research of materials and science which are cosmetic driven. ${ }^{1,2}$

With adhesive dentistry an impetus of aesthetic products came into the market that conservatively preens the tooth. One such product is a veneer: which are wafer-thin shells made out of dental ceramic that are bonded onto the front side of teeth. They are generally about 0.5 to $0.6 \mathrm{~mm}$ thick, which is about $1 / 2$ the thickness of a dime or twice the thickness of an eggshell. ${ }^{3}$ The present case series describes the rehabilitation of dislodged, discoloured restorations and irregular spacing in the maxillary anterior dentition with thin porcelain laminate veneers, to restore esthetics and function.

\section{Case Report 1}

A 26-year-old male patient visited the Department of Conservative Dentistry, DAV Dental College, Yamunanagar, Haryana. He complained of dislodged restoration of his front teeth and wanted to rehabilitate his aesthetic appearance (Fig. 1). By seeking patient's permission, a dental punctilio was carried out by taking a few photographs (smiling and talking), radiographs and making study models for the mock build up. Smile analysis was performed from the facial aspect, the bucco-lingual dimensions and finally the occlusal plane as per recommendations. ${ }^{4}$ This helped not only the clinician but the patient to anticipate the challenge ahead. The patient had a good periodontal health, with mild gingivitis for which oral prophylaxis was performed on the same visit. Also, there was no radiographic evidence of caries, thereby root canal therapy was not required. The patient's medical history did not reveal any systemic disease.

The treatment options were explored upon consultation. Options of composite and ceramic veneer were given. Since the patient's restoration had been dislodged twice before, he desired a more durable material indicating ceramic veneer.

\section{Treatment}

The first step in the treatment plan was restoring the missing tooth structure with composite. (Fig. 1) Shade selection was done by placing a button of shades on the adjacent moist surface of the tooth at cervical, middle and incisal third. The selected composite was A2 body, B1 enamel with clear translucent and opaque for the palatal shell. Bevel at $45^{\circ}$ along with starburst bevel was placed on enamel covering as much distance as the size of the missing tooth. After etching and bonding, placement of the material (Tetric $\mathrm{N}$ Ceram) was completed. Thereafter, laminate preparation was started. The tooth preparation for porcelain veneers must be uniform and, whenever possible, restricted entirely to the enamel. ${ }^{5}$ Local anaesthesia may be given if the preparation extends to dentin. The buccal surface of the tooth was prepared using three-tiered depth cutting burs of $0.3 \mathrm{~mm}$ dimension for rapid, adequate 
and conservative tooth reduction as per guidleines. ${ }^{6}$ It was moved from mesial to distal and by changing the angle $\left(45^{\circ}\right.$ for incisal half and $90^{\circ}$ for gingival half) to facilitate cutting in two planes. Facial reduction was achieved by reducing the labial contour till the depth of the grooves using a round end tapered bur and keeping contra-lateral tooth as a point of reference for adequate depth. A modified chamfer finish line with a bevel was placed to accommodate the adequate bulk. Once the facial reduction was achieved, preparation was carried to the proximal surface using a round tapered bur, without breakage of contact. Incisally wrap around preparation was done with a butt joint placed incisally or superior to the contact of the mandibular incisor in centric occlusion. Also, the tooth was reduced $2 \mathrm{~mm}$ incisally.

Finally, all angles were rounded with an extra fine, tapered-cylinder and round-end diamond bur. Silicone rubber and abrasive disk were used to smoothen the prepared surface (Fig. 2). ${ }^{5}$ Thereafter, retraction cord was placed in the gingival sulcus and a complete impression using double viscosity polyvinyl siloxane impression material was made just after the removal of retraction cord (Fig. 3). The patient was given temporaries till the final day of placement of veneer (Fig. 4).

\section{Luting of the veneer}

The temporary restoration was removed and the tooth was rinsed with water and pumice. The laboratory prepared veneers were then tried and the adaptation and occlusion was checked, which after confirmation was rinsed and dried. Ceramic was etched using 5\% hydrofluoric acid (IPS Empress ceramic etching gel, Ivoclar Vivadent) for $1 \mathrm{~min}$ to increase its surface energy. It was then rinsed using copious amount of water for $1 \mathrm{~min}$. The next step was silanization with $\mathrm{S}$ Monobond (Ivoclar Vivadent) and was gently air dried for $1 \mathrm{~min}$. Then a layer of bonding agent was applied and photopolymerized for 20 seconds.

To condition the tooth surface, polyester strips were first placed on the adjacent two teeth to protect them. The enamel was then activated with a slurry paste. After preparation, the enamel surface was etchedand-rinsed with $38 \%$ phosphoric acid (Ultradent, South Jordan, UT) for 30 seconds, which was followed by application of an adhesive (Excite, Ivoclar Vivadent). Adhesive cement was applied on the inner surface of the restorations before cementation.

After removal of excess cement from the buccal and palatal surfaces using an explorer and with the help of a mylar strip or a floss for the interproximal areas, a layer of glycerine was applied on the margins. The restorations were then photopolymerized and the occlusion was checked if any points came on the porcelain-tooth interface. Thereafter, the margins were finished and polished with diamond burs, rubber points and diamond polishing paste (Fig. 5). ${ }^{7}$

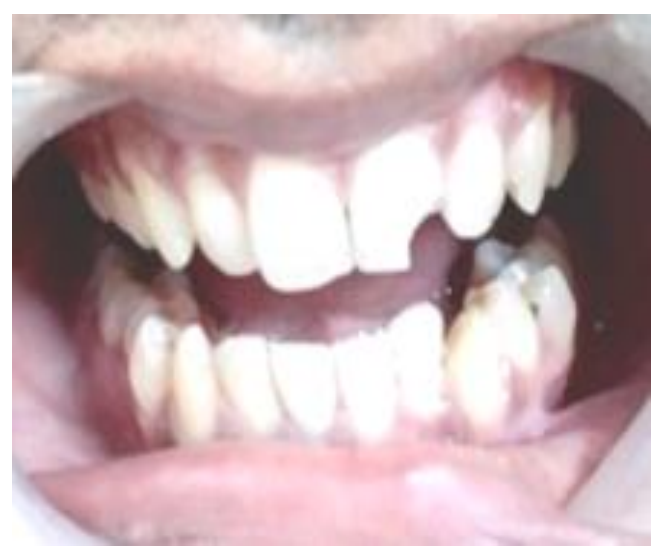

Fig. 1: Pre-operative intraoral photograph of case 1

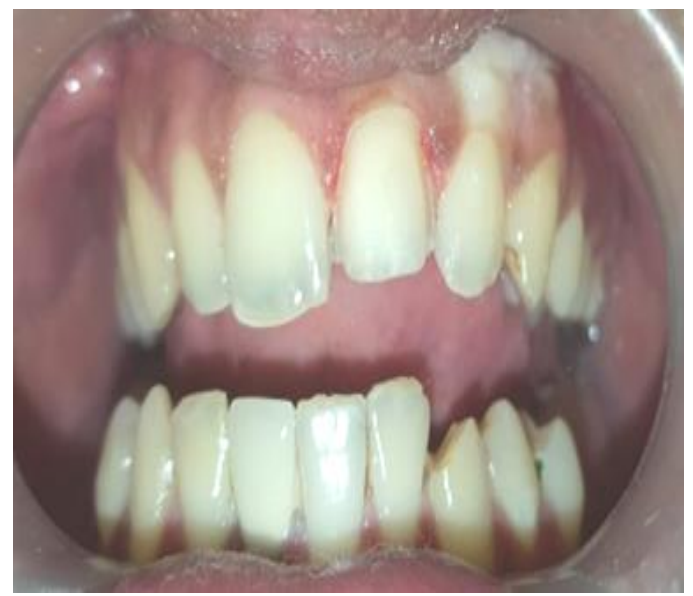

Fig. 2: Intraoral photograph showing Veneer preparation

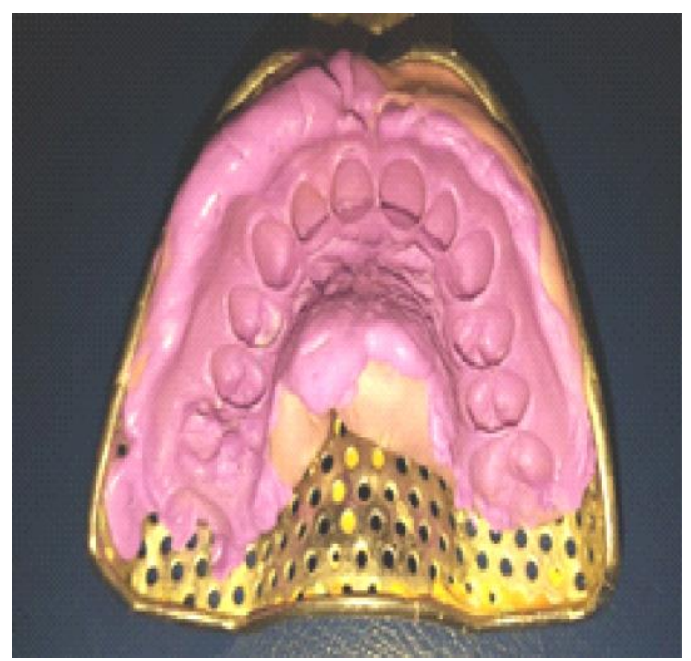

Fig. 3: Impression of the veneer preparation 


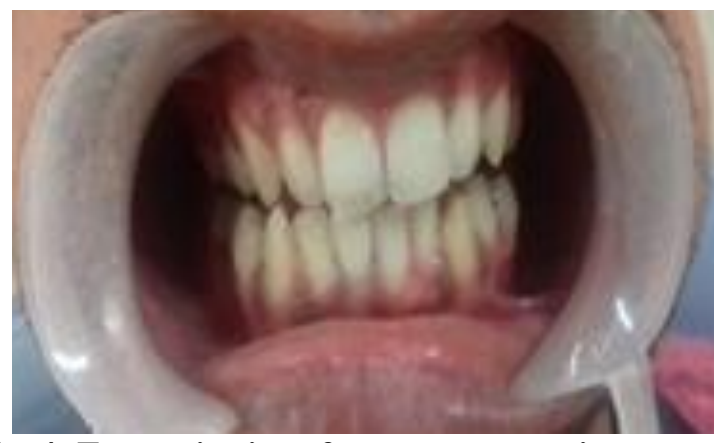

Fig. 4: Temporisation of veneer preparations

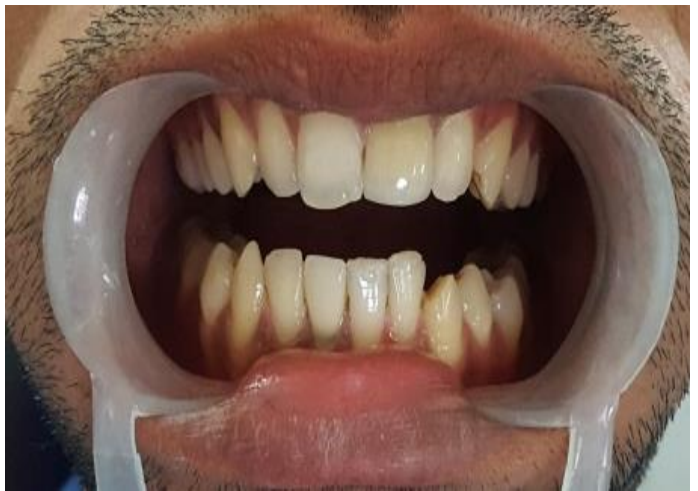

Fig. 5: Post-operative intraoral photograph showing veneer after cementation

\section{Case Report 2}

A 27 year old female patient reported to the department of Conservative Dentistry and Endodontics with the chief complaint of discoloured and dislodged restoration with respect to the upper front teeth. On clinical examination, the upper front teeth showed brown discrete discoloration due to previous restoration (Fig. 6). This case was similar to first case and composite restoration and ceramic veneers were planned. Veneer preparation and cementation was done as discussed earlier (Fig. 7).

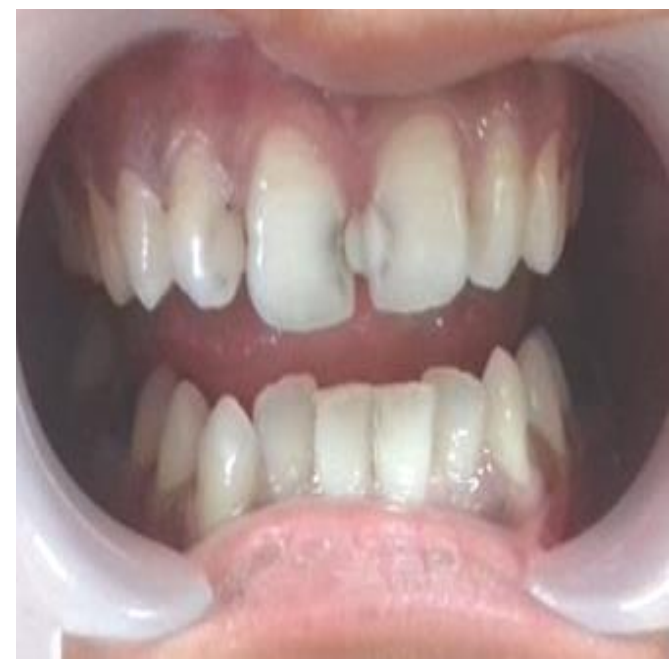

Fig. 6: Pre-operative photograph for the case 2

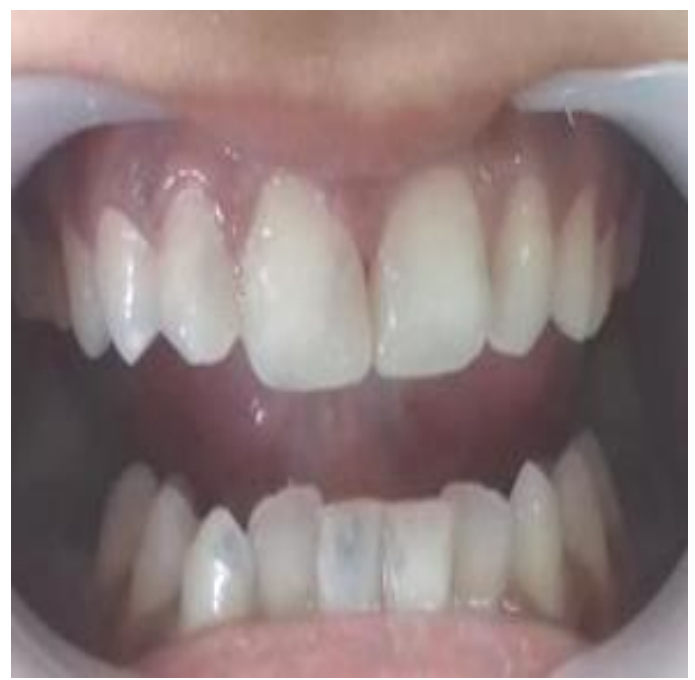

Fig. 7: Post-operative photograph for the case 2

\section{Case Report 3}

A 24 year old female patient reported to the Department of Conservative Dentistry and Endodontics with the chief complaint of irregular spaces with respect to upper front teeth. Clinical examination revealed irregular spaces between the maxillary central and lateral incisors on both sides (Fig. 8). Orthodontic treatment was advised but the patient refused because of esthetic reasons and requested for durable and short term treatment. Ceramic veneers were planned on 21 and 12. Before the treatment was started, mock-up was done to make the results predictable. Composite build up, veneer preparation and cementation were done according to the standard protocol as discussed before (Fig. 9).

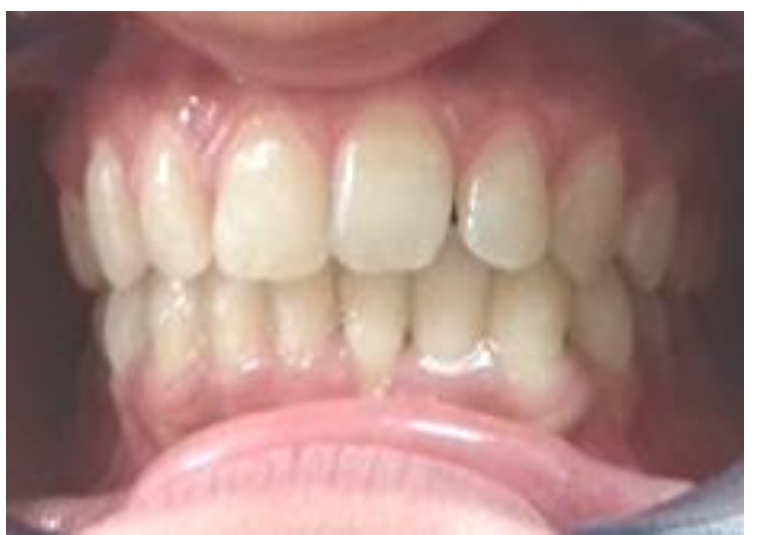

Fig. 8: Pre-operative photograph for the case 3 


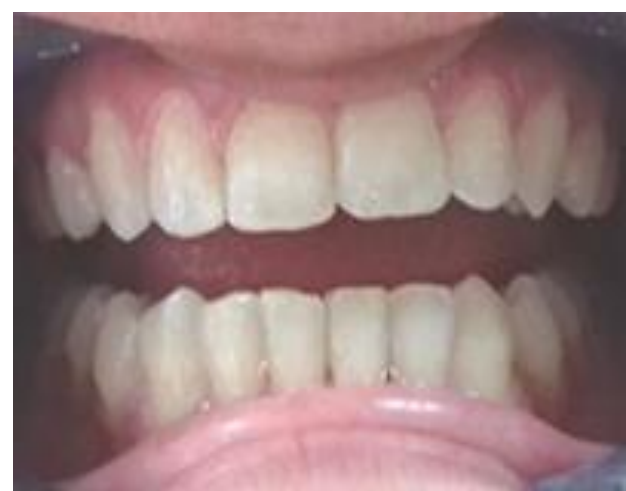

Fig. 9: Post-operative photograph for the case 3 with veneers on 11 and 21

\section{Discussion}

The use of ceramics in dentistry came about as an excellent long drawn solution to the requirements of a natural tooth like dental material..$^{6}$ It has been one of the most successful modalities of treatment that modern dentistry has to offer. ${ }^{4}$ To begin, the selection of restoration type is an important consideration. Taking into account that the patient wanted a conservative and a more durable restoration, composite and ceramics were considered. Although a composite restoration could have been applied, its long term prognosis remains questionable because of its susceptibility to discoloration, wear, and marginal fractures, thereby reducing the esthetic results in the long term. ${ }^{8}$ On the other hand, porcelain veneers are more durable anterior restorations with superior esthetics. ${ }^{9}$ Also, restrospective studies have stated success rates of veneers as high as $94 \%$ to $95 \% .^{4}$ According to Meijering and others, survival rates for veneer restorations were $94 \%$ for porcelain restorations, $90 \%$ for indirect composite restorations and $74 \%$ for direct composite restorations..$^{10}$ In yet another clinical study, direct resin composite veneers had a failure rate of $14 \%$ over 3 years of service, with a low incidence of marginal staining. ${ }^{11}$

The success of the treatment after the correct treatment modality, also depends on the adequate application of the techniques. In this case, bleaching was not required because there was no discolouration. Also, shade match for composite was done precisely because the final shade will be the combination of porcelain veneer, restored substrate and the luting agent. $^{12}$

Also, bond strength is affected by the type of adhesive used, therefore etch and rinse system was used for the bonding. But long-term study of porcelain veneers will be required in order to study their marginal integrity, marginal staining and their effect on gingival tissues.

\section{Clinical tips}

1. In case of diastema, proximal wrapping should be done.
2. Before photopolymerizng, a layer of glycerine should be applied to avoid creating an oxygen inhibition layer.

3. Final colour is a combination of porcelain veneer, luting agent and restored substrate.

4. Occlusion is never checked before the placement and final photopolymerization of veneer.

5. While placing the veneers, lights should be switched off with the overhead light on and directed towards the patient's toe.

6. While placing the luting cement, let the tip of syringe be in the cement till it reaches the other side. Retrieving it in between might produce a bubble, thereby affecting the final esthetics.

7. While placing multiple veneers, place all the units and then photopolymerize them together.

\section{Conclusion}

Modern day restorations offer great promise for expanded use of etched porcelain/ resin bonded system if the ability of bonding with dentin and enamel is respected and further researched. Although, in most cases, this treatment option results in longevity and patient satisfaction, the predictability of any restorative process will rest on the precise evaluation of occlusal conditions, knowledge of diagnosing and applicability of accurate technique for best result.

\section{Source of Funding: None.}

\section{Conflict of Interest: None.}

\section{References}

1. Goldstein RE. Change your smile. $4^{\text {th }}$ ed. USA: Quintessence Publication; 2009.

2. Beall AE. Can a new smile make you look more intelligent and successful? Dent Clin N Am 2007;51(2):289-97.

3. Porcelain Laminate Veneers. Available from https://www.animatedteeth.com/porcelain_veneers/t1_porcelain_veneers.htm. [Accessed 1 December 2018].

4. Gürel G. Porcelein laminate veneers: minimal tooth preparation by design. Dent Clin North Am 2007;51(2):419-31.

5. Rotoli BT, Lima DA, Pini NP, Aguiar FH, Pereira GD, Paulillo LA. Porcelain Veneers as an Alternative for Esthetic Treatment: Clinical Report. Oper Dent 2013;38(5):459-66.

6. Patil R, Shetty S. Esthetics with ceramics. In: Patil R. Esthetic Dentistry: An artist's science. Mumbai: PR publication, 2002; pp. 121-48.

7. Gresnigt M, Ozcan M. Esthetic rehabilitation of anterior teeth with laminates and sectional veneers. J Can Dent Assoc 2011;77:b143.

8. Peumans M, De Munck J, Fieuws S, Lambrechts P, Vanherle G, Van Meerbeek B. A prospective ten-year clinical trial of porcelain veneers. J Adhes Dent 2004;6(1):65-76.

9. Della Bona A.Bonding to ceramics: Scientific evidences for clinical dentistry In: Artes Médicas. Sao Paulo.UK: Scion Publishing; 2009. 
10. Meijering AC, Creugers NH, Roeters FJ, Mulder J. Survival of three types of veneer restorations in a clinical trial: a 2.5-year interim evaluation. $J$ Dent 1998;26(7):563-68.

11. Stappert CF, Ozden U, Gerds T, Strub JR. Longevity and failure load of ceramic veneers with different preparation designs after exposure to masticatory simulation. $J$ Prosthet Dent 2005;94(2):132-39.

12. Meyer Filho A, Vieira LC, Baratieri L, Lopes GC.

Porcelain veneers as an alternative for the esthetic treatment of stained anterior teeth: Clinical report. Quint Int 2005;36(3):191-96.

How to cite the article: Narula R, Dhir S, Gupta S, Gupta B, Patil N, Juneja M, Juneja S. Veneers in Esthetic Zone - A Case Series, J Dent Specialities 2018;6(2):177-181. 